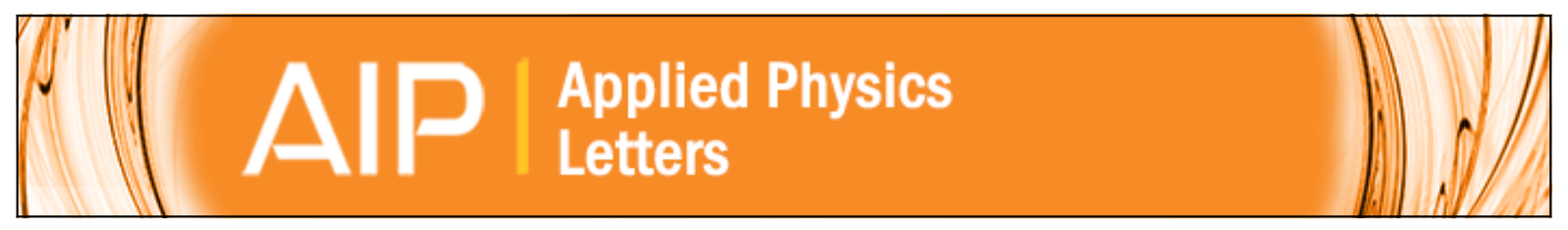

\title{
Three-dimensional left-handed material lens
}

R. V. Petrov, G. Srinivasan, M. I. Bichurin, and D. Viehland

Citation: Applied Physics Letters 91, 104103 (2007); doi: 10.1063/1.2778753

View online: http://dx.doi.org/10.1063/1.2778753

View Table of Contents: http://scitation.aip.org/content/aip/journal/apl/91/10?ver=pdfcov

Published by the AIP Publishing

\section{Over 700 papers \& presentations on multiphysics simulation}

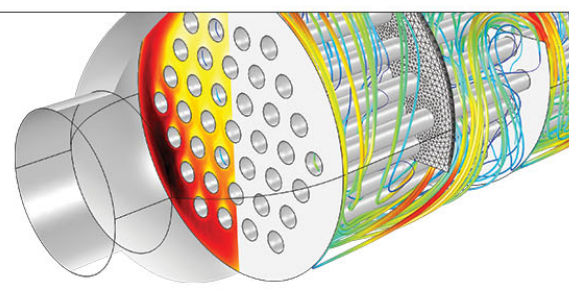




\title{
Three-dimensional left-handed material lens
}

\author{
R. V. Petrov and G. Srinivasan ${ }^{\text {a) }}$ \\ Department of Physics, Oakland University, Rochester, Michigan 48309 \\ M. I. Bichurin \\ Institute of Electronic and Information Systems, B. S. Peterburgskaya St. 41, \\ 173003 Veliky Novgorod, Russia \\ D. Viehland \\ Department of Materials Science and Engineering, Virginia Tech, Blacksburg, Virginia 24061
}

(Received 13 June 2007; accepted 12 August 2007; published online 5 September 2007)

\begin{abstract}
A model is provided for the image formation by a three-dimensional lens with negative index of refraction $(n)$ and compared with results for an array of split-ring resonators (SRRs) and wires. For $n=-1$, a linear decrease in image distance $r$ is expected with increasing object distance $l$. A nonlinear $r$ vs $l$ is anticipated for other $n$ values. A lens of an array of SRRs and wires was made and characterized over $1-18 \mathrm{GHz}$ and $n=-1$, and a gain of $\sim 8 \mathrm{~dB}$ at $11.25 \mathrm{GHz}$ was achieved. Results on $r$ vs $l$ are in excellent agreement with the model. (C) 2007 American Institute of Physics.
\end{abstract} [DOI: $10.1063 / 1.2778753$ ]

Veselago proposed the possibility of materials with a negative index of refraction $n$ and predicted that a planar slab having $n=-1$ could focus electromagnetic waves from a nearby source to an image on the opposite side of the slab. ${ }^{1}$ Since $n=\left(\mu_{r} \varepsilon_{r}\right)^{1 / 2}$, where $\mu_{r}$ and $\varepsilon_{r}$ are the relative permeability and permittivity, respectively, the objective is to achieve negative values of $\mu_{r}$ and $\varepsilon_{r}$. Pendry et al. showed that a two-dimensional array of thin wires exhibited negative $n .^{2}$ Shelby et al. used an array of split-ring resonators (SRRs) and wires to obtain negative values of $\mu_{r}$ and $\varepsilon_{r}$. ${ }^{3}$ Other significant developments include metamaterial wedges of rings and wires in a planar waveguide, ${ }^{4}$ free-space measurements on similar systems, ${ }^{5}$ observation of negative $n$ in yttrium vanadium oxide, ${ }^{6}$ and an array of gold nanorods at optical frequencies. ${ }^{7}$ Negative-index materials, also known as left-handed materials (LHM), are expected to show important phenomena including reversed geometric optics and reversed Doppler effect and could lead to a wide range of applications in miniature antenna, microwave and millimeter wave devices, imaging systems, and compact optics. ${ }^{2,8}$

This work is on (i) modeling of image formation by a three-dimensional LHM lens and (ii) verifications of the predictions with a lens of an array of SRRs and wires with $n=-1$ in the microwave region of the electromagnetic spectrum. In a recent study, a thin slab of SRR and wires was shown to exhibit multiple focal lengths at $10 \mathrm{GHz}$ by simply changing the source-lens distance. ${ }^{9}$ Here, we provide a model on focusing characteristics for a three-dimensional lens. Predictions on the focal length $r$ versus the source distance $l$ are discussed. A three-dimensional lens was then made with a $4 \times 4 \times 4$ array of SRRs and wires and characterized over $1-18 \mathrm{GHz}$. The structure was characterized in terms of $n$ vs $f$ and compared with theory. An index of refraction $n=-1$ was achieved for a frequency of $11.25 \mathrm{GHz}$. The focal length and the gain were measured as a function of the object distance. Data on focal length are found to be in very good agreement with the model.

First, we model the flow of microwave energy through an LHM slab of sides $d$ and width $h$, as shown in Fig. 1 . It is

\footnotetext{
${ }^{a)}$ Electronic mail: srinivas@oakland.edu
}

assumed that the width is large enough to enable focusing of microwaves from a source at a distance $l$. The refractive index $n=\sin \varphi / \sin \psi$. It can be shown by standard geometrical optics that $n$ in terms of lens dimensions and focal length $r$ is given by

$$
n=\sqrt{\frac{1+\frac{h^{2}}{4 d^{2}}\left(1+\frac{r}{l}\right)^{2}}{1+\frac{h^{2}}{4 l^{2}}}} .
$$

Figure 2 shows the anticipated lens characteristics from Eq. (1). Assuming $h=6 \mathrm{~cm}$ and $d=8 \mathrm{~cm}$, estimated $r$ as a function of $l$ is shown in Fig. 2(a) for negative $n$ values, for $-0.5<n<-2$. For $|n|<1$, one anticipates a nonlinear decrease in $r$ with increasing $l$. However, a linear decrease in $r$ with increasing $l$ is predicted for $n=-1$. For $|n|>1$, an increased $r$ is expected for increasing source distance $l$.

Next, assuming a lens with $n=-1, h=6 \mathrm{~cm}$, and $d=8 \mathrm{~cm}$, we considered the focusing behavior for several object distances. Figure 2(b) contains illustrations on focal length dependence on object distances, specifically for $l=2-8 \mathrm{~cm}$. For $l=2 \mathrm{~cm}$, for example, the focal length is estimated to be three times the object distance. For $l=4 \mathrm{~cm}$, the focal length is equal to the object distance. When $l$ is increased to $8 \mathrm{~cm}$, focusing cannot occur at all. Therefore, the radiation source must be located at a distance less than $d=8 \mathrm{~cm}$ for focusing to occur. Thus the results in Figs. 2(a)

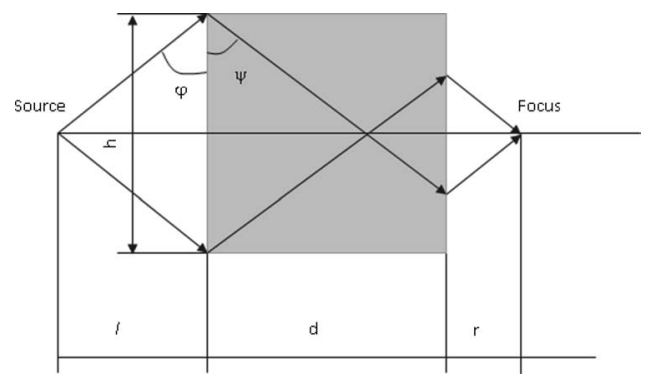

FIG. 1. Schematics showing refraction of microwaves through a lens with an index of refraction $n=-1$. The source is at a distance $l$ from the lens and the microwave is focused to a point at a distance $r$ from the lens. 
(a)

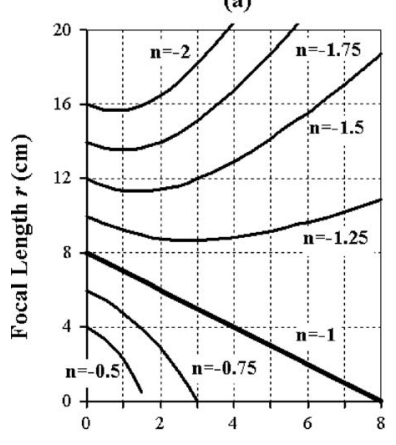

Object Distance $l(\mathrm{~cm})$
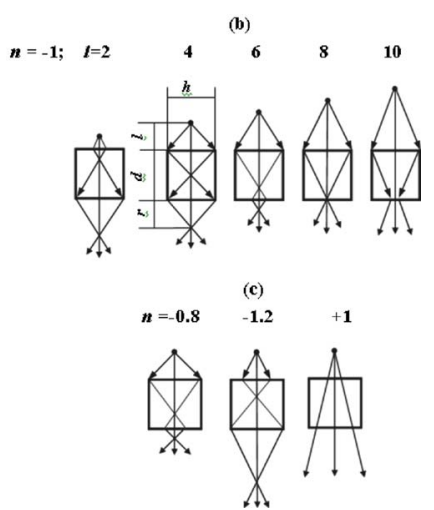

FIG. 2. (Color online) (a) Estimated focal length $r$ vs source distance $l$ as a function of the index of refraction $n$ for a lens of left-handed material (LHM). (b) Focusing characteristics of an LHM lens with thickness $d=6 \mathrm{~cm}$, width $h=8 \mathrm{~cm}$, and $n=-1$. The figures are for a series of object distances $l$. (c) Dependence of focal length $r$ on $n$.

and 2(b) for $n=-1$ imply $r+l=d$. The estimates in Fig. 2 are useful for designing a lens with proper dimensions for specific focal lengths. Results for a lens with $n=-1$ are of particular interest because of linear $r$ vs $l$.

Assuming that the lens geometry and its distance from a radiation source are constant, we next illustrate in Fig. 2(c) the effect of $n$ on the focal length $r$. On changing $n$ from -0.8 to -1.2 , the focal length is significantly increased, and when $n=1$ (i.e., medium with positive $\varepsilon_{r}$ and $\mu_{r}$ ), the propagating em waves are not refracted at all. Furthermore, for positive values of $n$, the distribution of em energy on exiting the lens is uniform. However, this will not be the case for an LHM lens; it is necessary to measure the em field distribution after transmission.

We then constructed a three dimensional lens array and performed microwave transmission measurements over the range of $1-18 \mathrm{GHz}$. It consisted of split-ring resonators (made with $0.2 \mathrm{~mm}$ diameter of $\mathrm{Cu}$ ) and straight wires $(1.8 \mathrm{~mm}$ long and $0.2 \mathrm{~mm}$ diameter of $\mathrm{Cu}$ ) bonded to a thin cardboard sheet $(\varepsilon \sim 1.2$ and loss tangent of $\sim 0.001)$, as shown in Fig. 3(a). The SRR and wire dimensions were chosen so that the resonance frequency will be $7.6 \mathrm{GHz}^{3} \mathrm{~A}$ lattice of such elements was constructed to form a $4 \times 4$ array in the $y z$ plane and stacked along the $x$ axis to form the lens shown in Fig. 3(b). The total dimensions of the LHM were $6 \times 8 \times 8 \mathrm{~cm}^{3}$. Microwave measurements were made with the radiation incident on the lens from frontal ( $z$ direction) or profile planes ( $x$ direction). A vector network analyzer (Agilent PNA) was used for microwave characterization. Two types of measurement systems were done: (i) power absorption and resonance frequency measurements using transmitting and receiving horns with the lens between (a)

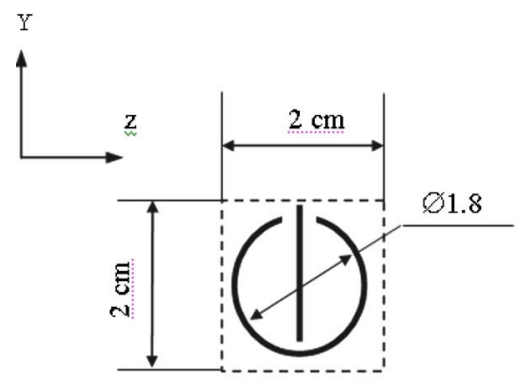

(b)

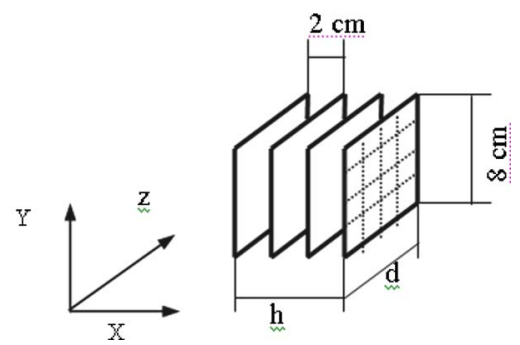

FIG. 3. (Color online) Schematics of a three-dimensional LHM array. (a) An LHM element of split-ring resonator (SRR) and straight wire. (b) $4 \times 4 \times 4$ array used in the experiment.

the two and (ii) measurements of field distribution, focal length, and refractive index using transmitting and receiving dipole antennas with the LHM lens mounted at their apertures.

Power transmission measurements are considered first. A relatively small insertion loss was measured for the incidence along $z$ direction, whereas a much larger loss was measured for $x$ incidence. Figure 4(a) shows data on power absorbed versus frequency profile for $z$ incidence. The maximum absorption of $30 \mathrm{~dB}$ occurs at $7.6 \mathrm{GHz}$. The absorption drops to a minimum value of $1.5 \mathrm{~dB}$ below $4 \mathrm{GHz}$ or above $11 \mathrm{GHz}$.
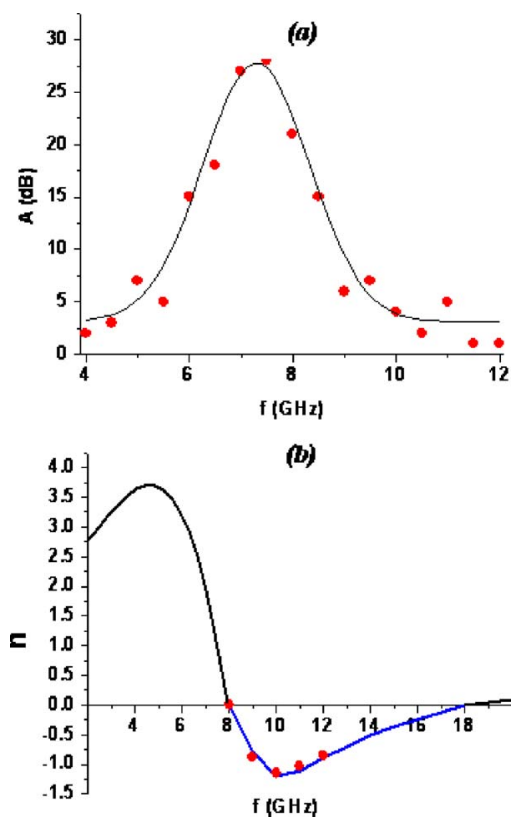

FIG. 4. (Color online) (a) Data on power absorption vs frequency for frontal (z) incidence for the LHM array. The solid line is guide to the eye. (b) Refractive index $n$ as a function of frequency for the array. The solid line represents theoretical values. The region of negative $n$ occurs at $8-18 \mathrm{GHz}$ where both $\varepsilon_{n}$ and $u_{r}$ are simultaneously negative. The circles are data points. 
For measurements on the lens properties, it is necessary to estimate the region of negative $n$. Since the resonance frequency is $7.6 \mathrm{GHz}$, one anticipates negative $n$ in this range of frequencies. Figure 4(b) shows calculated $n$ values over 2-18 GHz and was computed using the expressions for the permeability and permittivity of SRR and wires. The frequency dependence of permeability of SRR is given by ${ }^{6}$

$$
\mu^{\prime}=1+\frac{T_{m}^{2}\left(\omega_{m 0}-\omega\right) \omega_{m p}}{\left(\omega_{m 0}-\omega\right)^{2} T_{m}^{2}+1}
$$

where $\omega_{m 0}$ is the measured resonance frequency of SRR, $\omega_{m p}$ the magnetic plasma frequency, $T_{m}$ the relaxation time of the magnetic sublattice, and $\omega$ is the measurement frequency. The permittivity for the array of wires is given by ${ }^{6}$

$$
\varepsilon^{\prime}=1+\frac{T_{e}^{2}\left(\omega_{e 0}-\omega\right) \omega_{e p}}{\left(\omega_{e 0}-\omega\right)^{2} T_{e}^{2}+1},
$$

where $\omega_{e 0}$ is the measured resonance frequency of the wire lattice, $\omega_{e p}$ the electric plasma frequency, and $T_{e}$ the relaxation time for the electric sublattice. To find the unknown parameters in Eqs. (2) and (3), it was necessary to construct separate structures with SRR and with wires that were similar to the lens in terms of dimensions. Microwave transmission measurements were made with the SRR-only and wiresonly structures to find frequency edge of forbidden region. The data were then used to estimate $\omega_{m p}$ and $\omega_{e p}$.

Figure 4(b) shows estimated $n$ vs $f$ obtained using Eqs. (2) and (3) and the following measured values $f_{m 0}=f_{e 0}=7.6 \mathrm{GHz}$ and estimated values $f_{m p}=f_{e p}=11.8 \mathrm{GHz}$, $T_{m}=0.046$, and $T_{e}=0.14$. The results demonstrate that the material is expected to behave as an LHM in the frequency range of $8-18 \mathrm{GHz}$. At other frequencies, it behaves as a right-handed material. Note that $n=-1$ may be obtained in various ways: for example, $\varepsilon_{r}=\mu_{r}=-1, \varepsilon_{r}=-0.5$ and $\mu_{r}=$ -2 , or $\varepsilon_{r}=-2$ and $\mu_{r}=-0.5$. In Fig. 4, estimated $n$ are compared with the data that were obtained from the measurements of focal length $r$ as a function of source distance $l$, as will be described later. These parameters were then used in Eq. (1) to caluculate $n$ vs $f$ and are compared with theory. There is excellent agreement between data and theory in Fig. 4(b).

The lens was found to have $n=-1$ at $11.25 \mathrm{GHz}$ from power absorption measurements and theoretical and experimental determinations of $n$. The lens characteristics were measured in detail for this specific frequency. Measurements of the em field distribution exiting the LHM lens at $11.25 \mathrm{GHz}$ were measured as a function of the source distance $l$. The objective was to measure the focal length $r$ for comparison with the predictions in Fig. 2 and the lens gain. Representative results of such measurements are shown in Fig. 5. The transmitted power was measured in each point of $x z$ plane (for $y=4 \mathrm{~cm}$ ) for frontal incidence to obtain a twodimensional map. Measurements were realized in two steps by measuring the field strengths without and with the lens in place. The parameter $A$ in Fig. 5 is the difference in microwave power between these two cases. Data for source distances of $l=2$ and $6 \mathrm{~cm}$ are shown. The figure shows contours (in decibel) of the em field distribution that is transmitted through the lens. Light colored regions are the areas with the maximum amplitude of the field and the dark regions are the ones with the lowest magnitudes of the microwave field. For $l=6 \mathrm{~cm}$, one observes a rather sharp focus
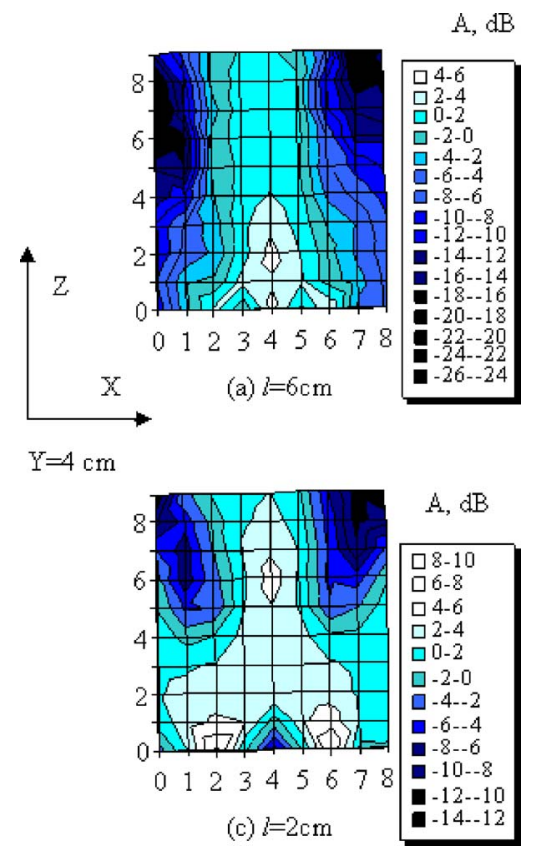

FIG. 5. (Color online) Profiles at $11.25 \mathrm{GHz}$ showing the focusing characteristics of the lens with $n=-1$ and for frontal incidence. The dark regions indicate a field reduction and the light regions indicate a microwave field amplification.

at $2 \mathrm{~cm}$ from the lens and a gain of $6 \mathrm{~dB}$ from the focal point. Upon moving the object closer to the lens, $l=2 \mathrm{~cm}$, focusing occurs at $6 \mathrm{~cm}$ from the lens and the gain is enhanced to $8 \mathrm{~dB}$. It is clear from the profiles that the focal point is well defined in space for the lens and that with increasing $l$, the focal length is decreased. These observations are in agreement with the predicted behavior of Fig. 1(b).

In conclusion, a model is discussed for focusing characteristics of a thick LHM lens. Estimates are provided for focal length variation with the source distance and the index of refraction. A three-dimensional array of split ring resonators and wires was constructed and characterized in the microwave frequencies. Based on the data and simulations, the lens has $n=-1$ over a narrow frequency range and the sum of the source distance $l$ and focal length $r$ equals the array dimension along the direction of incidence of microwaves. A gain of $\sim 8 \mathrm{~dB}$ at $11.25 \mathrm{GHz}$ has been achieved. The results are also important for the possible use of the lens as antenna element microwave devices.

This work was supported by grants from Oakland University ICR funds.

${ }^{1}$ V. G. Veselago, Usp. Fiz. Nauk 92, 517 (1964); Sov. Phys. Usp. 10, 509 (1968).

${ }^{2}$ J. B. Pendry, A. Holden, W. Stewart, and I. Youngs, Phys. Rev. Lett. 76, 4773 (1996).

${ }^{3}$ R. A. Shelby, D. R. Smith, and S. Schultz, Science 292, 77 (2001).

${ }^{4}$ A. A. Houck, J. B. Brock, and I. L. Chuang, Phys. Rev. Lett. 90, 137401 (2000).

${ }^{5}$ K. Li, S. J. McLean, R. B. Greegor, C. G. Parazzoli, and M. H. Tanielian, Appl. Phys. Lett. 82, 2535 (2003).

${ }^{6}$ R. A. Shelby, D. R. Smith, S. C. Nemat-Nasser, and S. Shultz, Appl. Phys. Lett. 78, 489 (2001).

${ }^{7}$ M. I. Bichurin, A. S. Tatarenko, G. Srinivasan, and J. V. Mantese, in Magnetoelectric Interaction Phenomena in Crystals, NATO Advance Studies Institute, Series B: Physics, edited by M. Fiebig, V. V. Eremenko, and I. E. Chupis (Kluwer, London, 2004), Vol. 164, p. 81-86.

${ }^{8}$ R. W. Ziolkowski and A. D. Kipple, IEEE Trans. Antennas Propag. 51, 2626 (2003).

J. D. Wilson and Z. D. Schitatiz, Appil. Fhys. Lete. 86, 021113 (2005). do to IP. 